\title{
Professional-amateur programs at Chungbuk National University
}

\author{
Y. Kim and J.-N. Yoon \\ Department of Astronomy and Space Science, \\ Chungbuk National University Observatory, \\ Chugbuk National University, Cheongju, 28644, Korea (E-mail: \\ ykkim153@chungbuk.ac.kr)
}

Received: November 18, 2019; Accepted: January 7, 2020

\begin{abstract}
Efforts at the Chungbuk National University, Korea, to encourage amateur astronomers to participate in astronomical observation are presented. The Chungbuk National University Observatory opened the Jincheon station with $1.0-\mathrm{m}$ and $0.6-\mathrm{m}$ optical telescopes for research and education. The observatory is equipped with a fully automated observation and control system. Since its opening in 2008, the observatory has developed amateur astronomer programs and provided training in astronomical observation. The observatory also aims at stimulating other observatories in Korea to engage in reserach and education. We summarize our programs and future plans regarding professional-amateur collaborations in Korea.
\end{abstract}

Key words: small telescopes - professional-amateur programs

\section{Introduction}

Small telescopes are recognized as useful observational tools, even in the current era of large professional telescopes. Many astronomers are using small telescopes to monitor variable or transient objects because of ease of installation of small instruments and lack of heavy competition for telescope time. Even though in Korea there are many small telescopes at universities, research institutes, astronomical observatories, and science high schools, these telescopes often remain underutilized.

The professional-amateur program can be understood as encouraging amateur astronomers to participate in astronomical observing. Amateur astronomers are in need of proper training to conduct observations, therefore educators are essential for the success of the program. Small telescopes at observatories can be used as training facilities for this purpose.

Chungbuk National University (CBNU) has a department of astronomy and space science where many astronomical educators are trained to help amateurs using small telescopes, and a university observatory (CBNUO) providing the technical support for various astronomical observations. Before explaining what 
is done at CBNU, the current status of small telescopes in Korea will be introduced. We will then discuss projects that can be done using small telescopes and will close by discussing the future of small telescopes in Korea.

\section{Current status of small telescopes in Korea}

The locations of facilities with small telescopes in Korea are shown in Fig. 1. Small telescopes are located in 12 education and science research institutes, 6 science museums, 28 astronomical observatories, 7 private observatories, and 8 universities. The Korean government funded the construction of many astronomical observatories since 2005 with the goal of increasing science literacy in Korea. Among these telescopes, there are 17 refractors and 40 reflectors. Reflectors with diameters larger than $50 \mathrm{~cm}$ are most common in the Korean observatories, and we must note that few of these telescopes are being used for research purposes.

About 5000 amateur astronomers are enrolled in the Korea Amateur Astronomer Society (KAAS), and $30 \%$ of its members are school teachers. However, most of them are more interested in astrophotography than photometry or spectroscopy. Because there are many amateur astronomers who have a small telescope with a diameter larger than $40 \mathrm{~cm}$ diameter, there is the potential of their engagement in scientific projects.

\section{Projects at CBNU}

The Department of Astronomy and Space Science at Chungbuk National University (CBNU) recognized this situation and began to teach students how to encourage amateur astronomers to participate in making astronomical observations. The department introduced graduate courses for public astronomy in 2005 , and now over 80 students with bachelor's, master's or Ph.D. degrees are working in astronomical observatories, science museums, and private astronomical institutes. The training of educators for public astronomy is being carried in connection with the University Observatory (CBNUO), which plays the role of technical supporter for other observatories as well as conducting active observations.

In the graduate program of public astronomy, 11 students have graduated with a Master's degree and one with a Ph.D. so far, while 19 students are enrolled in the Master's program, 6 students in the doctoral program, and one student in the integration program. Over 80 students who graduated in our department are active at various astronomical observatories, science museums, and private astronomical institutes.

CBNUO installed a 1-m Ritchey-Chrétien telescope in the Jincheon station of the university observatory in 2008. We began to observe variable stars with this telescope. One 0.6-m wide-field telescope has also been available since 2009 


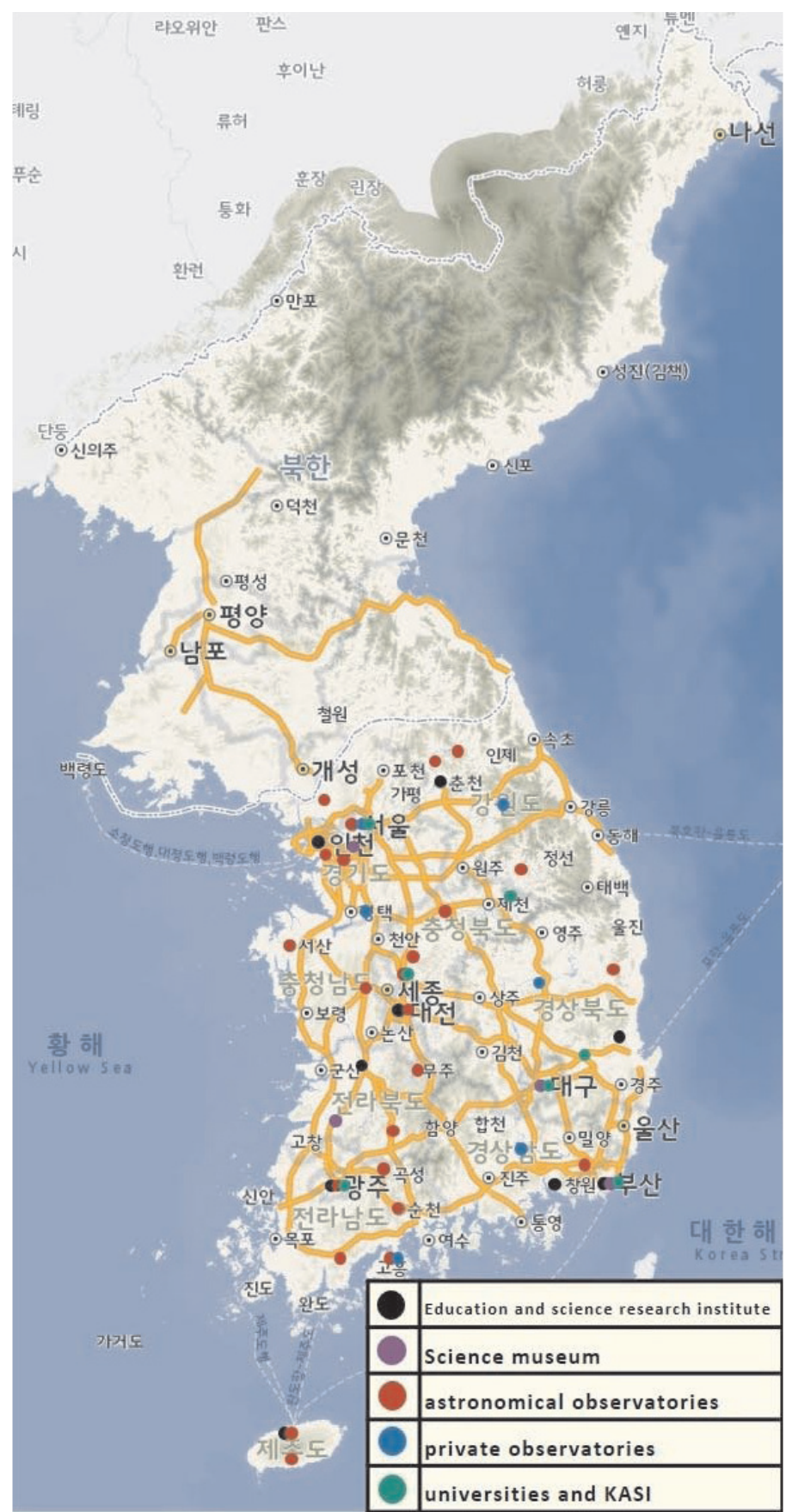

Figure 1. Distribution of small telescopes in Korea. 
and is now used as the main telescope for scientific observations. CBNUO developed a fully automated robotic observation program and control system for its 1-m, 0.6-m and 0.4-m telescopes for scientific observations.

As an example, the current status of the 0.6-m telescope is shown in Fig. 2. Between 2010 and 2018, observations were made on a total of 700 days, on average 5 hours per day and 100 days per year. A total of 141 objects were observed in the last 10 years. The maximum number of observing nights, 110, was for the magnetic cataclysmic variable DO Dra, and the maximum number of individual observations $(11,361)$ was for the eclipsing binary DY Lyn. Eclipsing binaries are analyzed by Professor Chun-Hwey Kim (see, for example, Kim et al., 2014). Today about 20 refereed papers concerning binaries have been published. Other objects such as exoplanets, asteroids, and satellites, are also observed. Hinse et al. (2015) and Wang et al. (2019) are papers that include exoplanet data obtained with CBNUO telescopes.

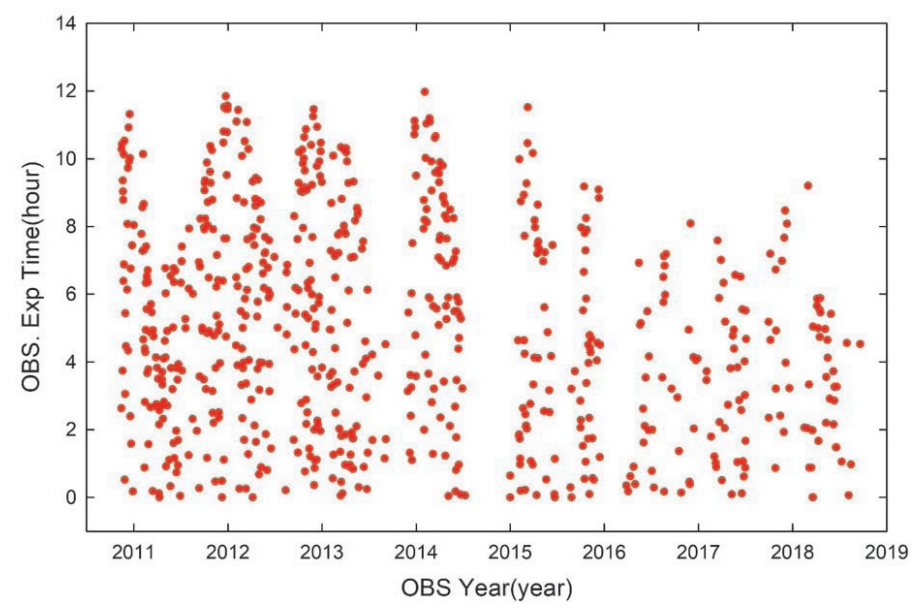

Figure 2. Observation status of the 0.6-m telescope of CBNUO.

CBNUO is also providing the know-how to other astronomical observatories related to the development of control and observational systems, as well as telescope maintenance. We are proud to have carried out 17 technical service projects for other astronomical observatories over the past 10 years. Yoon et al. $(2012,2013)$ are papers showing our efforts for setting up automatic observation. In order to encourage teachers to observe variable stars, we helped construct a $50-\mathrm{cm}$ telescope with a remote observation system at Gyeonggi Science High School in 2015, and we encouraged one of the teachers to observe variable stars. Many students are now being trained to observe variable stars with this telescope. However, it needs to be mentioned that it is very hard to convince high 
school teachers to observe variable stars. As amateur astronomers, most teachers are more interested in photography.

\section{CBNU future plans}

As described in the previous section, CBNUO accumulated the know-how about fully automatic observational systems as well as educating students who will later work in public astronomy, and to encourage amateur astronomers to carry out scientific observations. CBNU has taken proper measures to connect amateur astronomers with scientific data taking. Many alumni of our department who are now working in public astronomy are facilitating doing this.

Fortunately, the optical and IR astronomy division of the Korean Astronomical Society (KAS) is preparing a small telescope network project that will encourage amateur astronomers to participate in astronomical observations. Telescopes between $40 \mathrm{~cm}$ and $1 \mathrm{~m}$ diameter will be networked for common use in research and education. This small telescope network project, planned for 2020, will be competing for various research projects that either require a rapid response or require long-term, steady monitoring. Examples are observations of exoplanet transits, active white dwarf binary systems, solar system small bodies, supernovae, and Active Galactic Nuclei.

CBNU focuses on producing experts who will encourage amateur astronomers to participate in astronomical observing, and on providing efficient technical support for observing with small telescopes. The small telescope network brings small telescopes in full operation and offers astronomers competitive observational resources in several astronomical regions. Amateur astronomers will be encouraged to engage in international cooperation.

\section{Concluding remarks}

Small telescopes are very useful for researching interesting celestial objects, even in the era of big telescopes. CBNU is making efforts with CBNUO to help astronomical institutes develop fully automatic observational systems and maintain their telescopes, as well as to educate students to become experts to teach general astronomy and scientific observation. Such efforts will also provide amateur astronomers to take part in the scientific enterprise.

The interest of amateur astronomers in scientific observations will be amplified by the planned networking of small telescopes in Korea and assistance with the maintenance of small telescopes. Through their training at CBNU, students become experts and crucial assets in establishing professional-amateur programs in astronomy. 


\section{References}

Hinse, T.C., Han, W., Yoon, J.-N., Lee, C.-U., Kim, Y.-G., Kim, C.-H.: 2015, JASS 32, 21

Kim, C.-H., Song, M.-H., Yoon, J.-N., Han, W., Jeong, M.-J.: 2014, Astrophys. J. $\mathbf{7 8 8}, 134$

Wang, Y.-H., Wang, S., Hinse, T.C., Wu, Z.-Y., Davis, A.B., Hori, Y., Yoon, J.-N., Han, W., Nie, J.-D., Liu, H.-G., Zhang, H., Zhou, J.-L., Wittenmyer, R.A., Peng, X.-Y., Laughlin, G.: 2019, Astron. J. 157, 82

Yoon, J.-N., Lee, Y.S., Kim, C.-H., Kim, Y., Yim, H.-S., Han, W., Jeong, J.H.: 2012, JASS 29, 397

Yoon, J.-N., Kim, Y., Kim, D.-H., Yim, H.-S.: 2013, JASS 30, 327 